\title{
Invasive Lobular Breast Cancer as a Distinct Disease: Implications for Therapeutic Strategy
}

Jocelyn Luveta · Ruth M. Parks • David M. Heery · Kwok-Leung Cheung •

Simon J. Johnston

Received: November 18, 2019 / Published online: December 24, 2019

(c) The Author(s) 2019

\section{ABSTRACT}

Invasive lobular carcinoma comprises $10-15 \%$ of all breast cancers and is increasingly recognised as a distinct and understudied disease compared with the predominant histological subtype, invasive ductal carcinoma. Hallmarks of invasive lobular carcinoma include E-cadherin loss, leading to discohesive morphology with cells proliferating in single-file strands and oestrogen receptor positivity, with favourable response to endocrine therapy. This review summarises the distinct histological and molecular features of invasive lobular carcinoma with focus on diagnostic challenges and the impact on surgical management and

Enhanced Digital Features To view enhanced digital features for this article go to https://doi.org/10.6084/ m9.figshare.11358614.

J. Luveta $\cdot$ S. J. Johnston ( $₫)$

Division of Cancer and Stem Cells, Nottingham Breast Cancer Research Centre, School of Medicine, University of Nottingham, Nottingham, UK

e-mail: simon.johnston@nottingham.ac.uk

J. Luveta · D. M. Heery · S. J. Johnston Gene Regulation and RNA Biology, School of Pharmacy, University of Nottingham, Nottingham, UK

R. M. Parks · K.-L. Cheung Division of Medical Sciences and Graduate Entry Medicine, Nottingham Breast Cancer Research Centre, School of Medicine, University of Nottingham, Nottingham, UK medical therapy. Emphasis is placed on recent advances in our understanding of the unique molecular biology of lobular breast cancer and how this is optimising our therapy approach in the clinic.

Keywords: Aromatase inhibitors; Breast cancer; Endocrine therapy; Histology; Immunotherapy; Invasive lobular carcinoma; Molecular biology; Surgical management; Tamoxifen; Targeted therapy

\section{Key Summary Points}

This review provides a summary of the distinct features of lobular breast cancer and how these impact current clinical decision-making

An overview of the key diagnostic, surgical and medical therapy challenges in lobular breast cancer is presented

Key clinical trials of targeted and immunomodulatory therapies in lobular breast cancer are outlined

The article emphasises the functional implications of lobular breast cancer's unique molecular biology and their potential impact on therapy in the future 


\section{INTRODUCTION}

Invasive lobular breast cancer (ILC) accounts for $10-15 \%$ of all breast cancers making it the second most common type of breast cancer behind invasive ductal carcinoma (IDC, also known as invasive carcinoma of no special type) [1-4]. There has been an apparent increase in the incidence of ILC over the last 2 decades due to diagnostic advances $[5,6]$. Increased incidence of ILC is associated with the use of hormone replacement therapy in postmenopausal patients [7-9]. Compared with IDC, ILC tends to occur in older women [10].

Many studies show that ILC is associated with a good prognostic phenotype, i.e., well-differentiated (low grade), low-to-moderate proliferation index, hormone receptor positivity for both oestrogen receptor- $\alpha$ (ER) and progesterone receptor (PR), human epidermal growth factor receptor 2 (HER2)-negativity and basal marker negative status $[11,12]$. Although the majority of ILC is ERpositive and responds well to endocrine therapy, retrospective clinical cohort studies show that long-term outcomes of ILC are equivalent or even inferior to that of IDC [13-15]. However, the evidence is mixed and there are conflicting data to suggest that adjusting for stage would abolish any difference in long-term clinical outcome between ILC and IDC [16]. This highlights the need for further research into the biological basis of observed differences between ILC and IDC, coupled with prospective clinical trials aimed at improving outcomes for patients with ILC.

This article summarises the morphological and molecular features of ILC at presentation and how these affect the diagnosis and management of ILC. Current management strategy, key clinical trials and future directions as a result of functional study of the consequences of its unique molecular profile are discussed in this article.

\section{DISCUSSION}

\section{Histopathological and Molecular Characteristics of Lobular Breast Cancer}

Most ILC is classified as luminal A in terms of its molecular subtype: ER positive, PR positive, low proliferation index (Ki67) and HER2 negative $[11,12]$. In ILC, the basal subtype (triple negative: ER, PR and HER2 negative) occurs less frequently and is linked to a distinct "pleomorphic" histological subtype. Classic ILC is made up of uniform cells with spherical nuclei and indistinct nucleoli, whereas pleomorphic ILC tends to consist of larger cells with expansive eosinophilic cytoplasm [17].

The classic hallmark of ILC, found in 85-95\% of cases, is loss of E-cadherin protein expression, which leads to its distinctive discohesive appearance and characteristic single-file pattern of invasive cells (see Fig. 1) [11, 18, 19]. E-cadherin is a calcium-dependent transmembrane protein that mediates intercellular adhesion and polarity by homophilic attachment to adjacent cells; thus, loss of E-cadherin results in loss of intercellular adhesion with detached cells undergoing programmed cell death by anoikis [18, 20-22].

Mechanisms of E-cadherin loss in ILC include mutation or methylation of the $\mathrm{CDH} 1$ gene (located at chromosome 16q22.1) and loss of heterozygosity in the chromosome region 16q [23-25]. Another mechanism of E-cadherin loss is dysregulated expression of catenin-binding proteins $(\alpha, \beta, \gamma$ and p120-catenin), which anchor E-cadherin to the membrane and the actin cytoskeleton [26, 27]. Downregulation of catenin-binding protein (CBP) leads to its cytoplasmic redistribution and makes ILC cells resistant to anoikis. CBP downregulation also leads to activated Rho/Rock signalling, which

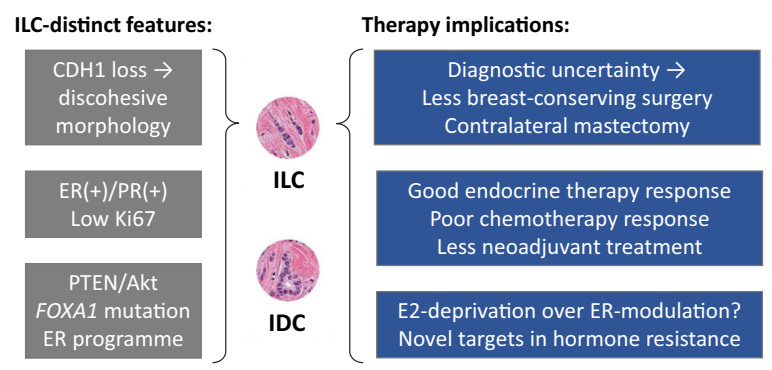

Fig. 1 Comparison of key molecular and histological features of ILC and IDC and the implications for treatment of ILC. E2 = oestradiol; PTEN/Akt refers to PTEN loss and Akt activation; ER programme refers to the distinct ER-mediated gene expression programme in ILC. Adapted with permission from Ciriello et al. [12] 
promotes cell migration $[22,26,27]$. These observations suggest that CBP downregulation enables ILC cells to survive and proliferate in their characteristic single-file pattern.

Loss of functional E-cadherin may be associated with epithelial-to-mesenchymal transition (EMT), although this remains controversial. Cadherin switching in CDH1-deficient tumours to $\mathrm{N}$-cadherin may favour a mesenchymal phenotype. However, since E-cadherin loss in ILC tends to be an early event, the association of EMT with later stages of cancer progression suggests that E-cadherin loss is not sufficient to cause EMT. This topic has been extensively described by McCart Reed and colleagues [11, 28].

Besides E-cadherin loss, ILC is enriched versus IDC for inactivating phosphatase and tensin homologue (PTEN) alterations (14\% vs. 3\%), forkhead box protein A1 (FOXA1) mutations (7\% vs. $2 \%)$ and runt-related transcription factor 1 (RUNX1) mutations (10\% vs. 3\%) [12]. Loss of the tumour suppressor, PTEN, as a result of homozygous chromosomal loss at 10q23, is associated with increased Akt phosphorylation, which is strongly activated in ILC, upregulates EGFR and thus creates a more aggressive phenotype [12, 29-31]. Along with enrichment of FOXA1 mutation, ILC is distinguished from IDC by relatively low frequency of GATA3 mutations (5\% vs. 20\%) [12]. FOXA1 and GATA3 are key transcriptional regulators of ER activity $[32,33]$. These findings suggest critical and mutually exclusive roles for FOXA1 and GATA3 in the evolution of ILC and IDC, respectively.

FoxA1 is a pioneer transcriptional factor that opens condensed chromatin to allow ER to bind at specific sites on the DNA, thereby inducing cell cycle progression and tumour growth $[32,34]$. FOXA1 is a component of a gene expression signature associated with luminal breast tumours, which is associated with a better prognosis [35]. FOXA1 mutations in ILC cluster in the forkhead (DNA-binding) domain $[12,36]$. This implies that mutations may affect the pioneer function of FoxA1 and thereby alter ligand-dependent ER binding to DNA. FOXA1 mutations may therefore alter the response to ER-targeted therapies such as tamoxifen, a selective ER modulator. For example, forkhead box mutations in FOXA1 may alter the avidity of FoxA1 binding to DNA at specific genome loci, thereby reprogramming genomic ER binding and leading to altered gene expression profiles associated with proliferation and/or endocrine resistance [36]. These hypotheses warrant urgent preclinical and clinical investigation.

\section{Diagnosis of Lobular Breast Cancer}

ILC does not always present with a firm lump in the breast and therefore poses particular challenges for its detection clinically and/or via screening [37]. Signs of ILC may include a thickened or swollen area in the breast with or without a change in the nipple shape (e.g., inverted nipple) and dimpling of the skin [38]. Relative to other subtypes of breast cancer, the extent of a primary ILC lesion is difficult to assess both clinically and by mammography because of its infiltrative growth pattern into the stroma without desmoplastic reaction $[38,39]$.

Due to its diffuse and discohesive ILC morphology, ILC cannot be accurately assessed by mammography alone [40]. Sensitivity of mammography for ILC ranges from 57 to $81 \%$ $[40,41]$. Furthermore, whilst magnetic resonance imaging (MRI) offers improved resolution, studies suggest that the ability of MRI to assess suitability for breast-conserving surgery is often suboptimal in cases of ILC [42]. In addition, compared with other subtypes, there is evidence that synchronous contralateral (i.e., bilateral) primary disease occurs more frequently in ILC $(20.9 \%$ in ILC versus $11.2 \%$ in IDC; $p<0.0001$ ) [43].

Late-presenting ILC has a distinct profile of distant metastatic sites. In ILC, transcoelemic spread leads to relative increase in frequency of gastrointestinal tract, peritoneal and ovarian metastases [13, 43, 44]. ILC that has spread to the gastrointestinal tract may present with gastrointestinal upset and bowel symptoms, including subacute and acute obstruction, while peritoneal disease may present with ascites, hydronephrosis and/or hydroureter. In terms of the 'classic' sites of breast cancer metastases, 
there is relatively more metastasis to bone and skin and less to lungs and brain [43, 44]. In patients on adjuvant therapy for ILC, or those being followed up post-adjuvant therapy, knowing the histological subtype is crucial in predicting the spread and monitoring for recurrence.

\section{Current Treatment of Primary Lobular Breast Cancer}

Current treatment for ILC follows identical treatment protocols as for all other histological subtypes of breast cancer [45]. This section focuses on how known ILC-specific features may impact surgical and medical management (i.e., endocrine therapy and chemotherapy). Emerging therapy modalities such as immunomodulation-already a mainstay of treatment in advanced breast cancer-are likely to have an impact on primary treatment protocols in the near future [46]. Immunotherapy is discussed further in "Future Treatment of Lobular Breast Cancer").

\section{Surgery}

In most cases of breast cancer, breast-conserving surgery by wide local excision is performed to remove the tumour with a margin of the surrounding normal tissue [45]. However, in ILC, there is evidence that $17-65 \%$ of patients have to undergo a second surgical intervention [47]. This raises concerns about the accuracy of ILC assessment due to its diffuse and multifocal morphology. Consequently, patients with ILC are more likely to undergo breast removal by mastectomy. Whilst offering greater resolution, MRI scanning may also increase false-positive detection of regional disease leading to an inappropriate increase in rates of mastectomy [42]. Following surgery, patients with ILC often undergo contralateral mastectomy because of the known tendency for bilateral ILC to occur [48].

Despite concerns regarding the uncertainty of complete excision of primary ILC following breast-conserving surgery, evidence suggests that modern surgical approaches confer the same rates of local recurrence for ILC, IDC and mixed histology tumours [49]. Furthermore, evidence shows that mastectomy may not offer a long-term survival advantage over breastconserving surgery [50]. These observations imply that the rate of breast-conserving surgery in ILC could be increased without compromising long-term clinical outcomes.

\section{Neoadjuvant Therapy}

Besides initial surgery, there is an option to have primary systemic treatment to manage ILC $[45,51]$. Neoadjuvant chemotherapy is aimed at improving rates of breast-conserving surgery. However, consensus has emerged that ILC tends to respond poorly to chemotherapy, leading to lower disease-free and overall survival following neoadjuvant chemotherapy compared with IDC [52-54]. Histological evidence indicates that ILC persists in up to $99 \%$ of cases (i.e., only $1 \%$ rate of pathological complete response) following anthracycline-based neoadjuvant chemotherapy [55]. The poor response to chemotherapy is also associated with its luminal A subtype (ER/PR positive, low mitotic index/Ki67) [53]. Neoadjuvant chemotherapy is generally not considered for patients with ILC as it is unlikely to improve rates of breast-conserving surgery but exposes patients to toxicities that can be life threatening.

As most ILC is luminal A type and hormone responsive, treatment with endocrine therapy in the neoadjuvant setting may be preferable to chemotherapy. A retrospective study of 61 patients with ER-positive ILC found that neoadjuvant letrozole reduced tumour size by a mean of $66 \%$ at 3 months, and the rate of successful breast conservation was $81 \%$ [56]. This small study demonstrates that there is untapped potential to increase rates of breast-conserving surgery and reduce rates of mastectomy in ILC-especially as endocrine therapy is optimised by combination with targeted therapies such as CDK4/6 inhibitors. Specific targeted therapies in ILC are discussed further in "Future Treatment of Lobular Breast Cancer".

\section{Adjuvant Therapy}

Post-surgery, depending on prognostic risk factors and biomarker expression, patients may 
need to undergo adjuvant radiotherapy, chemotherapy and/or endocrine therapy [45]. In high-risk cases of primary disease in which patients receive adjuvant chemotherapy, there is evidence that ILC has an inferior prognosis than IDC, even after correcting for confounding factors such as patient age and breast cancer stage [13]. This is corroborated by studies showing that taxane-based chemotherapy is less effective in patients with hormone receptor positive, low proliferation and/or lobular histologic subtype tumours $[57,58]$. On the other hand, studies have consistently shown that ILC responds well to endocrine therapy. In the adjuvant setting, tamoxifen, a selective ER modulator, is indicated for pre-menopausal women, whilst post-menopausal women are given a third-generation aromatase inhibitor such as anastrozole or letrozole, irrespective of the histological subtype of breast cancer (see [59] for a recent review).

There is retrospective clinical evidence that patients with ILC benefit less from adjuvant tamoxifen than patients with IDC (see Fig. 2). At 8 years of follow-up, disease-free survival on adjuvant tamoxifen was $66 \%$ for patients with ILC versus $75 \%$ for patients with IDC $(p=0.03)$ [60]. In contrast, benefit from adjuvant letrozole at 8 years was identical between ILC and IDC (82\% disease-free survival for both). This implies an intrinsic resistance to tamoxifen in ILC and that patients with ILC should be preferentially offered adjuvant aromatase inhibition. A current pre-surgery window-ofopportunity study (Clinical Trials registration no. NCT02206984) is investigating which ERtargeted therapy (fulvestrant vs. anastrozole vs. tamoxifen) is most effective in ILC [61]. With sufficient recruitment, this study could indicate which endocrine therapy agent gives the strongest signal of response in ILC.

There is evidence that adjuvant radiotherapy improves survival outcomes for patients with ILC [62-64]. However, relatively few studies have investigated radiotherapy in the specific context of ILC, and further study is urgently required.

\section{Future Treatment of Lobular Breast Cancer}

In the future, the treatment of ILC may be improved with better understanding and clinical application of its unique molecular profile. For example, there is evidence that amplification of fibroblast growth factor receptor 1 (FGFR1) is a feature specific to ILC $[65,66]$. A study by Sikora et al. found that the ILC cell line model, MM134, is intrinsically resistant to tamoxifen and provided evidence that this could be reversed by inhibition of FGFR1 [65]. The same in vitro study demonstrated that in ILC, ER drives a unique gene expression program associated with resistance to tamoxifen. Subsequently, the group showed that WNT4 (encoding a Wnt signalling pathway ligand) was the most strongly upregulated ER target gene in ILC and may represent a novel therapeutic target to enhance response to endocrine therapy in patients with ILC [67].

Acquired mutations in ESR1 are found in $5-25 \%$ of secondary breast tumours as a result of selective treatment pressure leading to constitutive ER activity [68-71]. Whilst ESR1 mutations are relevant to the choice of therapy in metastatic breast cancer (for example, using an ER downregulator, i.e., fulvestrant), there is no evidence that specific ESR1 mutations pre-exist in primary ILC [72]. However, the unique ERmediated gene expression programme in primary ILC leads to the hypothesis that alteration of ER transcriptional regulators-e.g., DNA binding domain mutations in FOXA1 and RUNX1-may link to endocrine resistance and clinical outcomes in an ILC-dependent manner. As a potential target in endocrine-resistant breast cancer, FoxA1 is therefore a promising future therapeutic research area for ILC [73-75]. Traditionally, transcription factors have been considered therapeutically intractable, although ligand-activated nuclear receptors such as ER are the classic counterexample.

Cyclin-dependent kinase (CDK) 4/6 inhibitors, e.g., palbociclib and ribociclib, are licensed first line in advanced ER-positive, HER2-negative breast cancer in combination with endocrine therapy $[76,77]$. Multiple clinical trials are investigating the use of CDK4/6 inhibitors in the neoadjuvant and adjuvant settings for ER-positive breast cancer. To better understand how ILC might respond to 
A

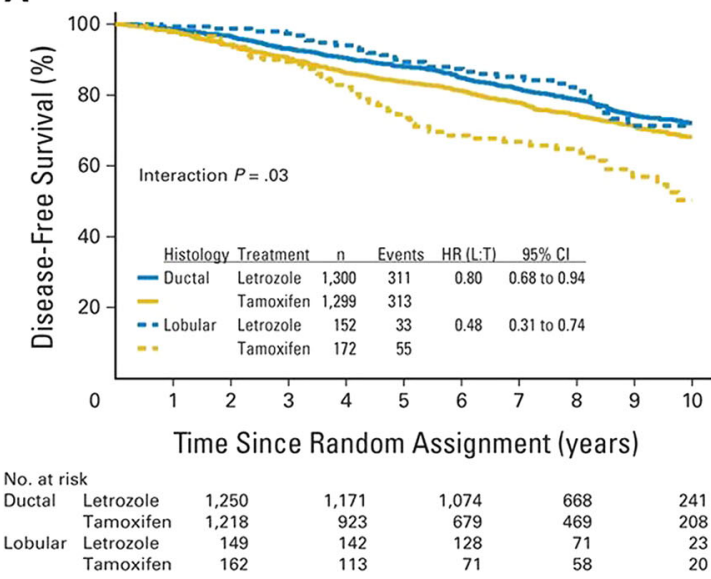

Fig. 2 Retrospective clinical evidence of a differential response to different classes of endocrine therapy in ILC. These data formed the basis of in vitro functional and early clinical studies of the differences between ILC and IDC. Kaplan-Meier plots show a disease-free survival and

neoadjuvant CDK4/6 inhibition, the Palbociclib and Endocrine Therapy for Lobular Breast Cancer Preoperative Study (PELOPS, Clinical Trials registration no. NCT02764541) is measuring the pathological complete response rate after 24 weeks of endocrine therapy with or without palbociclib [78]. Given the relative lack of chemotherapy response in ILC, this neoadjuvant trial (expected to complete in 2020) is potentially pivotal for patients with ILC. The trial design also incorporates a window-of-opportunity phase to test the hypothesis that letrozole is more effective in ILC than tamoxifen (see Fig. 3) [78].

Other strategies under clinical investigation for ILC include ROS1 targeting with crizotinib in advanced E-cadherin-negative, ER-positive lobular breast cancer and diffuse gastric cancer, known as the ROLO study (Clinical Trials registration no. NCT03620643) [79]. This trial exploits a synthetic lethality between E-cadherin deficiency and inhibition of the tyrosine kinase ROS1 [80]. Crizotinib is an existing ROS1 tyrosine kinase inhibitor licensed for use in ROS1-positive non-small-cell lung cancer that could in theory be repurposed for E-cadherindeficient cancers. If positive response rates are seen in advanced ILC, this approach may lead to further trials investigating ROS1 inhibition in the adjuvant or neoadjuvant settings.
B

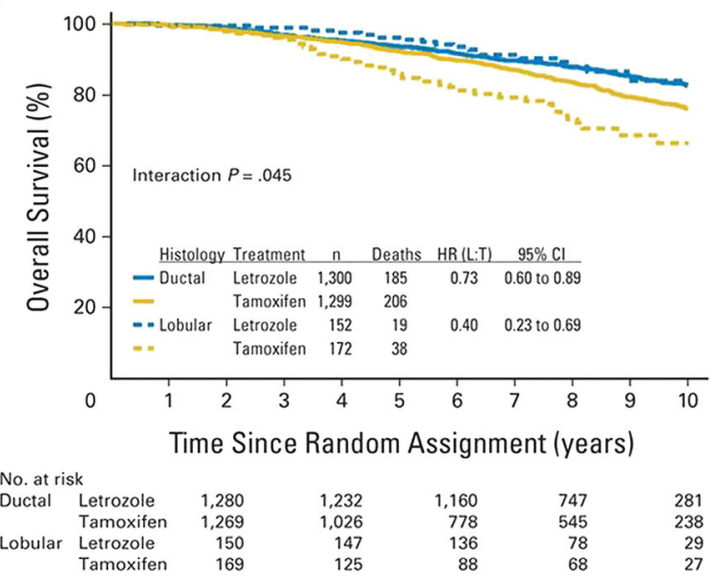

b overall survival stratified by histology (ductal/lobular) and endocrine therapy agent (letrozole, $\mathrm{L} /$ tamoxifen, $\mathrm{T}$ ); $N=2923$. Reproduced with permission from Metzger Filho et al. [60]

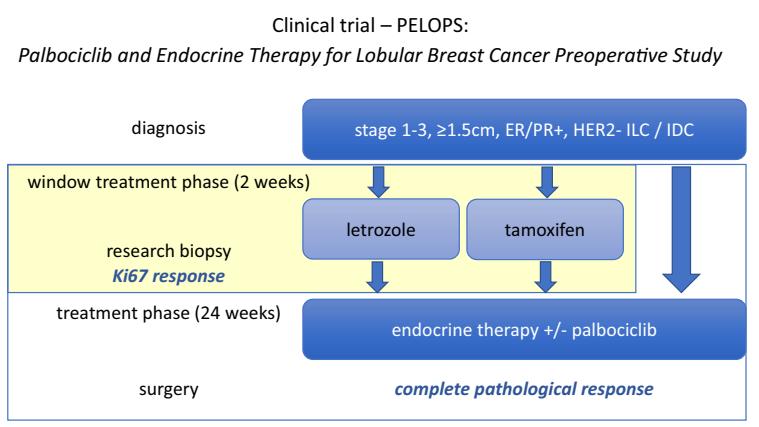

Fig. 3 PELOPS trial of neoadjuvant palbociclib, including a window-of-opportunity phase to provide prospective evidence on endocrine therapy of choice in ILC [78]. Study population is enriched for ILC. Treatment with palbociclib is randomly allocated. Primary outcome measure is complete pathological response

Recent evidence suggests that immune-related gene expression defines some ILC subtypes and is associated with survival rate $[12,81]$. Compared with IDC, there is evidence that ILC exhibits higher activity of almost all types of immune cells [81]. In this study by Du et al., evidence of lymphocytic infiltration in ILC tumours is linked to neoadjuvant chemotherapy response. However, a study by Desmedt et al. shows that even though lymphocyte infiltration is present in ILC, it is $21 \%$ lower than in IDC $(p<0.001)$ [82]. In ILC, high lymphocyte infiltration is associated with highly 
proliferative tumours, suggesting that there is a subgroup of ILC likely to respond well to existing immune checkpoint (PD-1, PD-L1 and CTLA4) inhibitors [82].

Response of ILC tumours to immune checkpoint inhibition is currently being investigated in a phase 2 clinical trial assessing the efficacy of carboplatin and atezolizumab in metastatic lobular breast cancer (the GELATO study, Clinical Trials registration no. NCT03147040) [83]. Atezolizumab is a monoclonal antibody targeting programmed death ligand 1 (PD-L1). Its mechanism of action is to disrupt the T cell inhibitory interaction of PD-L1 (on antigen-presenting cells) with its receptor PD-1 (on T-cells), thereby "taking the brakes off" the immune response. The study also aims to find novel markers of response to carboplatin plus atezolizumab, which could be very informative for future trials of immunotherapy in ILC.

\section{CONCLUSION}

ILC has distinct morphological features that relate to key molecular hallmarks such as loss of E-cadherin, which can make diagnosis and surgical treatment more challenging compared with IDC. On the other hand, ILC is characterised by molecular features such as ER and PR positivity that are associated with good clinical outcome and response to endocrine therapy.

The challenge for the field remains to improve our understanding of the functional implications of the unique molecular profile of ILC, beyond loss of E-cadherin. Linking ILC-associated molecular features with clinical phenotype-including response to endocrine therapy-and developing novel therapeutic strategies for biomarker-led and immunomodulatory clinical trials will be key to improving long-term outcomes for patients with ILC.

\section{ACKNOWLEDGEMENTS}

Funding. No funding or sponsorship was received for this study or publication of this article.
Authorship. All named authors meet the International Committee of Medical Journal Editors (ICMJE) criteria for authorship for this article, take responsibility for the integrity of the work as a whole, and have given their approval for this version to be published.

Disclosures. All authors (Jocelyn Luveta, Ruth M. Parks, David M. Heery, Simon J. Johnston) have nothing to disclose. Kwok-Leung Cheung is a member of the journal's editorial board but has nothing else to disclose.

Compliance with Ethics Guidelines. This article is based on previously conducted studies and does not contain any studies with human participants or animals performed by any of the authors.

Data Availability. Data sharing is not applicable to this article as no datasets were generated or analysed during the current study.

Open Access. This article is distributed under the terms of the Creative Commons Attribution-NonCommercial 4.0 International License (http://creativecommons.org/licenses/ by-nc/4.0/), which permits any noncommercial use, distribution, and reproduction in any medium, provided you give appropriate credit to the original author(s) and the source, provide a link to the Creative Commons license, and indicate if changes were made.

Open Access. This article is licensed under a Creative Commons Attribution-NonCommercial 4.0 International License, which permits any non-commercial use, sharing, adaptation, distribution and reproduction in any medium or format, as long as you give appropriate credit to the original author(s) and the source, provide a link to the Creative Commons licence, and indicate if changes were made. The images or other third party material in this article are included in the article's Creative Commons licence, unless indicated otherwise in a credit line to the material. If material is not included in the article's Creative Commons licence and your intended use is not permitted by statutory regulation or exceeds the permitted use, you 
will need to obtain permission directly from the copyright holder. To view a copy of this licence, visit http://creativecommons.org/licenses/by$\mathrm{nc} / 4.0 /$.

\section{REFERENCES}

1. Li CI, Anderson BO, Daling JR, Moe RE. Trends in incidence rates of invasive lobular and ductal breast carcinoma. JAMA. 2003;289(11):1421-4.

2. Martinez V, Azzopardi JG. Invasive lobular carcinoma of the breast: incidence and variants. Histopathology. 1979;3(6):467-88.

3. Li CI, Daling JR. Changes in breast cancer incidence rates in the United States by histologic subtype and race/ethnicity, 1995 to 2004. Cancer Epidemiol Biomark Prev. 2007;16(12):2773-80.

4. Lakhani SR, Ellis IO, Schnitt SJ, Tan PH, van de Vijver MJ. WHO classification of tumours of the breast. 4th ed. New Yok: WHO Press; 2012.

5. Li CI, Anderson BO, Porter P, Holt SK, Daling JR, Moe RE. Changing incidence rate of invasive lobular breast carcinoma among older women. Cancer. 2000;88(11):2561-9.

6. Biglia N, Mariani L, Sgro L, Mininanni P, Moggio G, Sismondi P. Increased incidence of lobular breast cancer in women treated with hormone replacement therapy: implications for diagnosis, surgical and medical treatment. Endocr Relat Cancer. 2007;14(3):549-67.

7. Newcomb PA, Titus-Ernstoff L, Egan KM, Trentham-Dietz A, Baron JA, Storer BE, et al. Postmenopausal estrogen and progestin use in relation to breast cancer risk. Cancer Epidemiol Biomark Prev. 2002;11(7):593-600.

8. Newcomer LM, Newcomb PA, Potter JD, Yasui Y, Trentham-Dietz A, Storer BE, et al. Postmenopausal hormone therapy and risk of breast cancer by histologic type (United States). Cancer Causes Control. 2003;14(3):225-33.

9. Kotsopoulos J, Chen WY, Gates MA, Tworoger SS, Hankinson SE, Rosner BA. Risk factors for ductal and lobular breast cancer: results from the nurses' health study. Breast Cancer Res. 2010;12(6):R106.

10. Colleoni M, Rotmensz N, Maisonneuve P, Mastropasqua MG, Luini A, Veronesi $P$, et al. Outcome of special types of luminal breast cancer. Ann Oncol. 2012;23(6):1428-36.
11. McCart Reed AE, Kutasovic JR, Lakhani SR, Simpson PT. Invasive lobular carcinoma of the breast: morphology, biomarkers and 'omics. Breast Cancer Res. 2015; 17:12.

12. Ciriello G, Gatza ML, Beck AH, Wilkerson MD, Rhie SK, Pastore A, et al. Comprehensive molecular portraits of invasive lobular breast cancer. Cell. 2015;163(2):506-19.

13. Adachi $\mathrm{Y}$, Ishiguro $\mathrm{J}$, Kotani $\mathrm{H}$, Hisada $\mathrm{T}$, Ichikawa $\mathrm{M}$, Gondo N, et al. Comparison of clinical outcomes between luminal invasive ductal carcinoma and luminal invasive lobular carcinoma. BMC Cancer. 2016;16:248.

14. Pestalozzi BC, Zahrieh D, Mallon E, Gusterson BA, Price KN, Gelber RD, et al. Distinct clinical and prognostic features of infiltrating lobular carcinoma of the breast: combined results of 15 International Breast Cancer Study Group clinical trials. J Clin Oncol. 2008;26(18):3006-14.

15. Rakha EA, El-Sayed ME, Powe DG, Green AR, Habashy H, Grainge MJ, et al. Invasive lobular carcinoma of the breast: response to hormonal therapy and outcomes. Eur J Cancer. 2008;44(1):73-83.

16. Wasif N, Maggard MA, Ko CY, Giuliano AE. Invasive lobular vs. ductal breast cancer: a stage-matched comparison of outcomes. Ann Surg Oncol. 2010;17(7):1862-9.

17. Butler D, Rosa M. Pleomorphic lobular carcinoma of the breast: a morphologically and clinically distinct variant of lobular carcinoma. Arch Pathol Lab Med. 2013;137(11):1688-92.

18. Rakha EA, Patel A, Powe DG, Benhasouna A, Green $\mathrm{AR}$, Lambros $\mathrm{MB}$, et al. Clinical and biological significance of E-cadherin protein expression in invasive lobular carcinoma of the breast. Am J Surg Pathol. 2010;34(10):1472-9.

19. Sinn HP, Kreipe H. A brief overview of the WHO classification of breast tumors, 4th edition, focusing on issues and updates from the 3rd edition. Breast Care (Basel). 2013;8(2):149-54.

20. Birchmeier $\mathrm{W}$, Behrens J. Cadherin expression in carcinomas: role in the formation of cell junctions and the prevention of invasiveness. Biochim Biophys Acta. 1994;1198(1):11-26.

21. Handschuh G, Candidus S, Luber B, Reich U, Schott $\mathrm{C}$, Oswald S, et al. Tumour-associated E-cadherin mutations alter cellular morphology, decrease cellular adhesion and increase cellular motility. Oncogene. 1999;18(30):4301-12.

22. Schackmann RC, van Amersfoort M, Haarhuis JH, Vlug EJ, Halim VA, Roodhart JM, et al. Cytosolic 
p120-catenin regulates growth of metastatic lobular carcinoma through Rock1-mediated anoikis resistance. J Clin Investig. 2011;121(8):3176-88.

23. Droufakou S, Deshmane V, Roylance R, Hanby A, Tomlinson I, Hart IR. Multiple ways of silencing E-cadherin gene expression in lobular carcinoma of the breast. Int J Cancer. 2001;92(3):404-8.

24. Sarrio D, Moreno-Bueno G, Hardisson D, SanchezEstevez C, Guo M, Herman JG, et al. Epigenetic and genetic alterations of APC and $\mathrm{CDH} 1$ genes in lobular breast cancer: relationships with abnormal E-cadherin and catenin expression and microsatellite instability. Int J Cancer. 2003;106(2):208-15.

25. Da Silva L, Parry S, Reid L, Keith P, Waddell N, Kossai M, et al. Aberrant expression of E-cadherin in lobular carcinomas of the breast. Am J Surg Pathol. 2008;32(5):773-83.

26. Dabbs DJ, Bhargava R, Chivukula M. Lobular versus ductal breast neoplasms: the diagnostic utility of p120 catenin. Am J Surg Pathol. 2007;31(3):427-37.

27. Dabbs DJ, Kaplai M, Chivukula M, Kanbour A, Kanbour-Shakir A, Carter GJ. The spectrum of morphomolecular abnormalities of the E-cadherin/catenin complex in pleomorphic lobular carcinoma of the breast. Appl Immunohistochem Mol Morphol. 2007;15(3):260-6.

28. McCart Reed AE, Kutasovic JR, Vargas AC, Jayanthan J, Al-Murrani A, Reid LE, et al. An epithelial to mesenchymal transition programme does not usually drive the phenotype of invasive lobular carcinomas. J Pathol. 2016;238(4):489-94.

29. Akbani R, Ng PK, Werner HM, Shahmoradgoli M, Zhang F, Ju Z, et al. A pan-cancer proteomic perspective on The Cancer Genome Atlas. Nat Commun. 2014;5:3887.

30. Cancer Genome Atlas N. Comprehensive molecular portraits of human breast tumours. Nature. 2012;490(7418):61-70.

31. Lau MT, Klausen C, Leung PC. E-cadherin inhibits tumor cell growth by suppressing PI3K/Akt signaling via beta-catenin-Egr1-mediated PTEN expression. Oncogene. 2011;30(24):2753-66.

32. Hurtado A, Holmes KA, Ross-Innes CS, Schmidt D, Carroll JS. FOXA1 is a key determinant of estrogen receptor function and endocrine response. Nat Genet. 2011;43(1):27-33.

33. Theodorou V, Stark R, Menon S, Carroll JS. GATA3 acts upstream of FOXA1 in mediating ESR1 binding by shaping enhancer accessibility. Genome Res. 2013;23(1):12-22.
34. Carroll JS, Liu XS, Brodsky AS, Li W, Meyer CA, Szary AJ, et al. Chromosome-wide mapping of estrogen receptor binding reveals long-range regulation requiring the forkhead protein FoxA1. Cell. 2005;122(1):33-43.

35. Perou CM, Sorlie T, Eisen MB, van de Rijn M, Jeffrey SS, Rees CA, et al. Molecular portraits of human breast tumours. Nature. 2000;406(6797):747-52.

36. Robinson JL, Holmes KA, Carroll JS. FOXA1 mutations in hormone-dependent cancers. Front Oncol. 2013;3:20.

37. Lopez JK, Bassett LW. Invasive lobular carcinoma of the breast: spectrum of mammographic, US, and MR imaging findings. Radiographics. 2009;29(1): 165-76.

38. Biglia N, Maggiorotto F, Liberale V, Bounous VE, Sgro LG, Pecchio S, et al. Clinical-pathologic features, long term-outcome and surgical treatment in a large series of patients with invasive lobular carcinoma (ILC) and invasive ductal carcinoma (IDC). Eur J Surg Oncol. 2013;39(5):455-60.

39. Kim SH, Cha ES, Park CS, Kang BJ, Whang IY, Lee AW, et al. Imaging features of invasive lobular carcinoma: comparison with invasive ductal carcinoma. Jpn J Radiol. 2011;29(7):475-82.

40. Helvie MA, Paramagul C, Oberman HA, Adler DD. Invasive lobular carcinoma. Imaging features and clinical detection. Investig Radiol. 1993;28(3): 202-7.

41. Butler RS, Venta LA, Wiley EL, Ellis RL, Dempsey PJ, Rubin E. Sonographic evaluation of infiltrating lobular carcinoma. AJR Am J Roentgenol. 1999;172(2):325-30.

42. Barker SJ, Anderson E, Mullen R. Magnetic resonance imaging for invasive lobular carcinoma: is it worth it? Gland Surg. 2019;8(3):237-41.

43. Arpino G, Bardou VJ, Clark GM, Elledge RM. Infiltrating lobular carcinoma of the breast: tumor characteristics and clinical outcome. Breast Cancer Res. 2004;6(3):R149-56.

44. Mathew A, Rajagopal PS, Villgran V, Sandhu GS, Jankowitz RC, Jacob M, et al. Distinct pattern of metastases in patients with invasive lobular carcinoma of the breast. Geburtshilfe Frauenheilkd. 2017;77(6):660-6.

45. National Institute for Health and Care Excellence, UK. Early and locally advanced breast cancer: diagnosis and management. 2018. Clinical Guideline NG101. Available online: https://www.nice. org.uk/guidance/ng101. Accessed Nov 2019 
46. Thompson ED, Taube JM, Asch-Kendrick RJ, Ogurtsova A, Xu H, Sharma R, et al. PD-L1 expression and the immune microenvironment in primary invasive lobular carcinomas of the breast. Mod Pathol. 2017;30(11):1551-60.

47. Christgen M, Steinemann D, Kuhnle E, Langer F, Gluz O, Harbeck N, et al. Lobular breast cancer: clinical, molecular and morphological characteristics. Pathol Res Pract. 2016;212(7):583-97.

48. Babiera G, Lowy AM, Davidson S, Singletary EE. The role of contralateral prophylactic mastectomy in invasive lobular carcinoma. Breast J. 1997;1(3):2-6.

49. Braunstein LZ, Brock JE, Chen YH, Truong L, Russo $\mathrm{AL}$, Arvold ND, et al. Invasive lobular carcinoma of the breast: local recurrence after breast-conserving therapy by subtype approximation and surgical margin. Breast Cancer Res Treat. 2015;149(2): 555-64.

50. Fodor J, Major T, Toth J, Sulyok Z, Polgar C. Comparison of mastectomy with breast-conserving surgery in invasive lobular carcinoma: 15-year results. Rep Pract Oncol Radiother. 2011;16(6):227-31.

51. Tubiana-Hulin M, Stevens D, Lasry S, Guinebretiere JM, Bouita L, Cohen-Solal C, et al. Response to neoadjuvant chemotherapy in lobular and ductal breast carcinomas: a retrospective study on 860 patients from one institution. Ann Oncol. 2006;17(8):1228-33.

52. Cocquyt VF, Blondeel PN, Depypere HT, Praet MM, Schelfhout VR, Silva OE, et al. Different responses to preoperative chemotherapy for invasive lobular and invasive ductal breast carcinoma. Eur J Surg Oncol. 2003;29(4):361-7.

53. Cristofanilli M, Gonzalez-Angulo A, Sneige N, Kau SW, Broglio K, Theriault RL, et al. Invasive lobular carcinoma classic type: response to primary chemotherapy and survival outcomes. J Clin Oncol. 2005;23(1):41-8.

54. Nagao T, Kinoshita T, Hojo T, Tsuda H, Tamura K, Fujiwara Y. The differences in the histological types of breast cancer and the response to neoadjuvant chemotherapy: the relationship between the outcome and the clinicopathological characteristics. Breast. 2012;21(3):289-95.

55. Brunello E, Brunelli M, Manfrin E, Nottegar A, Bersani S, Vergine M, et al. Classical lobular breast carcinoma consistently lacks topoisomerase-IIalpha gene amplification: implications for the tailored use of anthracycline-based chemotherapies. Histopathology. 2012;60(3):482-8.

56. Dixon JM, Renshaw L, Dixon J, Thomas J. Invasive lobular carcinoma: response to neoadjuvant letrozole therapy. Breast Cancer Res Treat. 2011;130(3):871-7.

57. Valero V. Primary chemotherapy with docetaxel for the management of breast cancer. Oncology (Williston Park). 2002;16(6 Suppl 6):35-43.

58. Valero V, Buzdar AU, McNeese M, Singletary E, Hortobagyi GN. Primary chemotherapy in the treatment of breast cancer: the University of Texas M. D. Anderson Cancer Center experience. Clin Breast Cancer. 2002;3(Suppl 2):S63-8.

59. Johnston SJ, Cheung KL. Endocrine therapy for breast cancer: a model of hormonal manipulation. Oncol Ther. 2018;6:141.

60. Metzger Filho O, Giobbie-Hurder A, Mallon E, Gusterson B, Viale G, Winer EP, et al. Relative effectiveness of letrozole compared with tamoxifen for patients with lobular carcinoma in the BIG 1-98 trial. J Clin Oncol. 2015;33(25):2772-9.

61. ClinicalTrials.gov. Endocrine response in women with invasive lobular breast cancer United States: National Library of Medicine; 2014 [Clinical trial]. https://clinicaltrials.gov/ct2/show/NCT02206984. Accessed Nov 2019

62. Diepenmaat LA, van der Sangen MJ, van de PollFranse LV, van Beek MW, van Berlo CL, Luiten EJ, et al. The impact of postmastectomy radiotherapy on local control in patients with invasive lobular breast cancer. Radiother Oncol. 2009;91(1):49-53.

63. Stecklein SR, Shen X, Mitchell MP. Post-mastectomy radiation therapy for invasive lobular carcinoma: a comparative utilization and outcomes study. Clin Breast Cancer. 2016;16(4):319-26.

64. Warneke J, Berger R, Johnson C, Stea D, Villar H. Lumpectomy and radiation treatment for invasive lobular carcinoma of the breast. Am J Surg. 1996;172(5):496-500.

65. Sikora MJ, Cooper KL, Bahreini A, Luthra S, Wang G, Chandran UR, et al. Invasive lobular carcinoma cell lines are characterized by unique estrogen-mediated gene expression patterns and altered tamoxifen response. Cancer Res. 2014;74(5): 1463-74.

66. Sikora MJ, Jankowitz RC, Dabbs DJ, Oesterreich S. Invasive lobular carcinoma of the breast: patient response to systemic endocrine therapy and hormone response in model systems. Steroids. 2013;78(6):568-75.

67. Sikora MJ, Jacobsen BM, Levine K, Chen J, Davidson NE, Lee AV, et al. WNT4 mediates estrogen receptor signaling and endocrine resistance in invasive 
lobular carcinoma cell lines. Breast Cancer Res. 2016;18(1):92.

68. Li S, Shen D, Shao J, Crowder R, Liu W, Prat A, et al. Endocrine-therapy-resistant ESR1 variants revealed by genomic characterization of breast-cancerderived xenografts. Cell Rep. 2013;4(6):1116-30.

69. Merenbakh-Lamin K, Ben-Baruch N, Yeheskel A, Dvir A, Soussan-Gutman L, Jeselsohn R, et al. D538G mutation in estrogen receptor-alpha: a novel mechanism for acquired endocrine resistance in breast cancer. Cancer Res. 2013;73(23):6856-64.

70. Robinson DR, Wu YM, Vats P, Su F, Lonigro RJ, Cao $\mathrm{X}$, et al. Activating ESR1 mutations in hormoneresistant metastatic breast cancer. Nat Genet. 2013;45(12):1446-51.

71. Toy W, Shen Y, Won H, Green B, Sakr RA, Will M, et al. ESR1 ligand-binding domain mutations in hormone-resistant breast cancer. Nat Genet. 2013;45(12):1439-45.

72. Desmedt C, Pingitore J, Rothe F, Marchio C, Clatot $F$, Rouas G, et al. ESR1 mutations in metastatic lobular breast cancer patients. NPJ Breast Cancer. 2019;5:9.

73. Johnston SJ, Carroll JS. Transcription factors and chromatin proteins as therapeutic targets in cancer. Biochim Biophys Acta. 2015;1855(2):183-92.

74. Johnston SJ, Holmes KA, Carroll JS. Targeting FoxA1 in estrogen receptor-positive breast cancer: biological characterization of kinase regulators. Cancer Res. 2015;76(14):S2906.

75. Nakshatri H, Badve S. FOXA1 as a therapeutic target for breast cancer. Expert Opin Ther Targets. 2007;11(4):507-14.
76. Im SA, Lu YS, Bardia A, Harbeck N, Colleoni M, Franke F, et al. Overall survival with ribociclib plus endocrine therapy in breast cancer. N Engl J Med. 2019;381(4):307-16.

77. Turner NC, Slamon DJ, Ro J, Bondarenko I, Im SA, Masuda N, et al. Overall survival with palbociclib and fulvestrant in advanced breast cancer. N Engl J Med. 2018;379(20):1926-36.

78. ClinicalTrials.gov. Palbociclib and endocrine therapy for lobular breast cancer United States: National Library of Medicine; 2016. https://clinicaltrials.gov/ ct2/show/NCT02764541.

79. ClinicalTrials.gov. ROS Targeting with crizotinib in advanced E-cadherin negative, ER positive lobular breast cancer or diffuse gastric cancer study United States: National Library of Medicine. https:// clinicaltrials.gov/ct2/show/NCT03620643.

80. Bajrami I, Marlow R, van de Ven M, Brough R, Pemberton HN, Frankum J, et al. E-cadherin/ROS1 inhibitor synthetic lethality in breast cancer. Cancer Discov. 2018;8(4):498-515.

81. Du T, Zhu L, Levine KM, Tasdemir N, Lee AV, Vignali DAA, et al. Invasive lobular and ductal breast carcinoma differ in immune response, protein translation efficiency and metabolism. Sci Rep. 2018;8(1):7205.

82. Desmedt C, Salgado R, Fornili M, Pruneri G, Van den Eynden G, Zoppoli G, et al. Immune infiltration in invasive lobular breast cancer. J Natl Cancer Inst. 2018;110(7):768-76.

83. ClinicalTrials.gov. Assessing efficacy of carboplatin and atezolizumab in metastatic lobular breast cancer Netherlands: National Library of Medicine; 2017. https://clinicaltrials.gov/ct2/show/NCT03147040. 